\title{
CoRa -A general approach for quantifying biological feedback control
}

\author{
Mariana Gómez-Schiavon ${ }^{1}$, Hana El-Samad ${ }^{1,2,3,4, *}$ \\ 1 Department of Biochemistry and Biophysics, University of California, San Francisco, San \\ Francisco, CA, USA. \\ 2 Cell Design Initiative, University of California, San Francisco, CA, USA \\ 3 Chan-Zuckerberg Biohub, San Francisco, CA, USA. \\ 4 Cell Design Institute, San Francisco, CA, USA.
}

\section{* Hana.El-Samad@ucsf.edu}

Short title: Quantifying biological feedback control with CoRa

\begin{abstract}
Feedback control is a fundamental underpinning of life, underlying homeostasis of biological processes at every scale of organization, from cells to ecosystems. The ability to evaluate the contribution and limitations of feedback control mechanisms operating in cells is a critical step for understanding and ultimately designing feedback control systems with biological molecules. Here, we introduce CoRa -or Control Ratio-, a general framework that quantifies the contribution of a biological feedback control mechanism to adaptation using a mathematically controlled comparison to an identical system that does not contain the feedback. CoRa provides a simple and intuitive metric with broad applicability to biological feedback systems.
\end{abstract}

Feedback control is a mechanism by which a system can assess its own state and use this information to react accordingly [14]. Cells and organisms make abundant use of feedback control [3], in particular negative feedback to deploy corrective actions. Negative feedback is instrumental in the ability of biological systems to restore homeostasis after a perturbation 5, 8, 10,16, a property known in engineering as disturbance rejection and in the biological sciences as adaptation. Despite the importance of feedback, no systematic and generalizable approaches exist to quantify the contribution of a negative feedback loop to adaptation in biological networks. Here, we propose CoRa -or Control Ratio-, a mathematical approach that tackles this problem. CoRa follows the classical notion of Mathematically Controlled Comparisons [1] by assessing the performance of a biological system with feedback control to a locally analogous system without feedback. The locally analogous system without feedback has identical structure and parameters to those of the feedback system, except for the feedback link, and both systems rest at the same steady-state value before the perturbation. As a result, the divergence in their behavior after they are challenged with a perturbation isolates and quantifies the contribution of the feedback control (Fig. 1). CoRa can be defined and computed for any biological system described by a solvable set of ordinary differential equations, irrespective of its complexity. CoRa can also be efficiently computed across different parameter values of a system, allowing a global view of the performance of its feedback under different conditions.

CoRa formalism. To apply CoRa, two systems are considered: the intact system that has the feedback structure, and a locally analogous system without feedback. Both systems have exactly the same biochemical reactions and parameters, with the only difference being the replacement of the feedback input in the locally analogous system with a constant input. The magnitude of this constant input is chosen to generate identical steady-state values for the output and all internal variables for both systems under a given parameter set $\Theta$. In order to evaluate the contribution of the feedback to adaptation following a perturbation in a specific parameter $\rho \in \Theta$, one can apply a small perturbation $\left(\rho \rightarrow \rho^{\prime}\right)$ and scrutinize the output of the two systems. If $Y$ is the output of the system with feedback and $Y_{N F}$ is that of the locally analogous system without feedback, then the contribution of the feedback to adaptation after this perturbation in $\rho \in \Theta$ can be quantified as the ratio of the response of the feedback system 
A
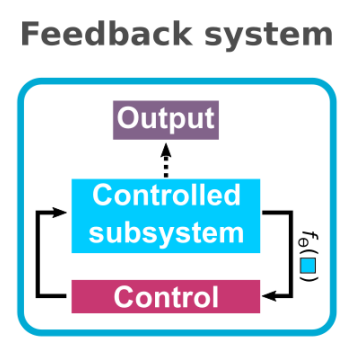

\section{Feedback system \\ - Locally analogous no-feedback system}

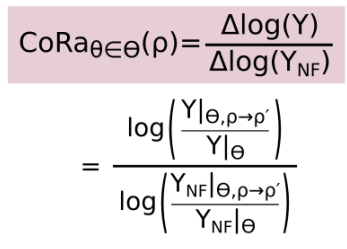

Locally analogous no-feedback system

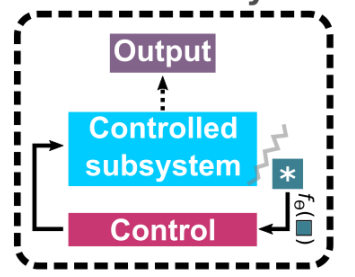

Small perturbation to $\rho$

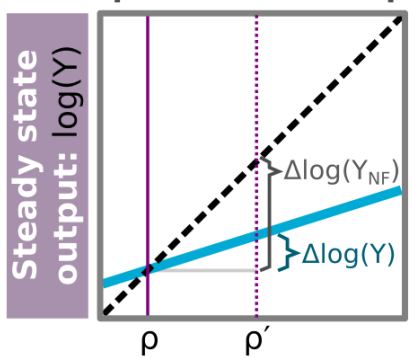

B
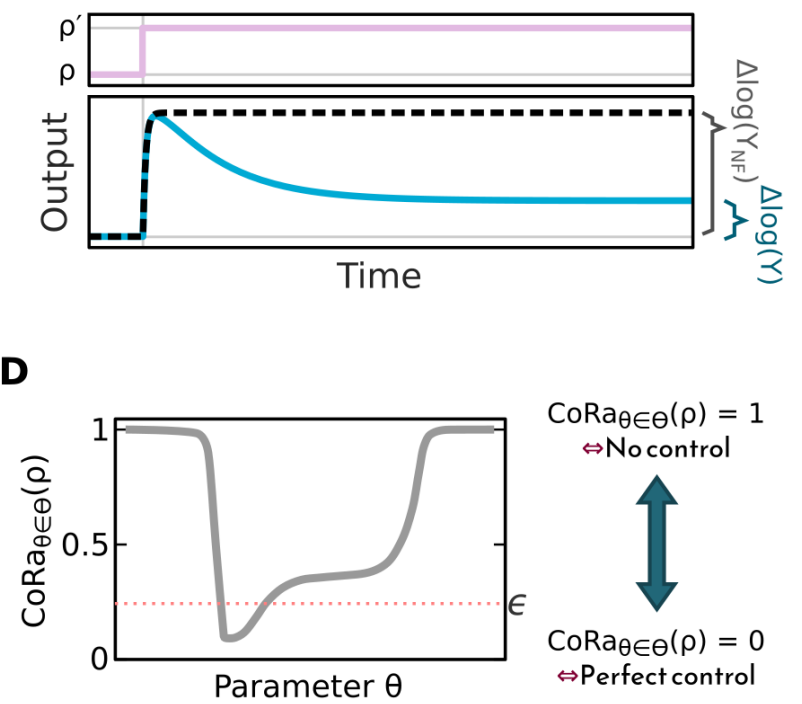

$\operatorname{CoRa}_{\theta \in \Theta}(\rho)=1$ $\Leftrightarrow$ No control

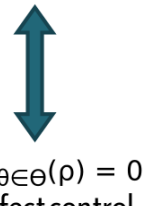

$\operatorname{CoRa}_{\theta \in \Theta}(\rho)=0$
$\Leftrightarrow$ Perfect control $^{-1}$

Figure 1. Explaining CoRa, Control Ratio approach. (A) Diagram of a system with feedback (left) and its locally analogous system without feedback (right). The controlled subsystem is the biological network to which feedback control is applied. For a given parameter set $(\Theta)$, the constant input is fixed such that the input signal $\left(f_{\Theta}\right)$ is the same between the feedback system and the locally analogous system without feedback. (B) Plot of the output of the system with feedback (blue line) and the locally analogous system (black dashed line) as functions of time after a small perturbation to a specific parameter $\left(\rho \rightarrow \rho^{\prime}\right.$, with $\left.\rho \in \Theta\right)$. Since both systems are identical before the perturbation, any differential output response reflects the properties of the feedback. $Y$ is used to denote output of system with feedback and $Y_{N F}$ the output of locally analogous system with no feedback. (C) Definition of CoRa. For each parameter set $\Theta$, the CoRa value for perturbation to $\rho, \operatorname{CoRa}_{\theta \in \Theta}(\rho)$, is defined as the ratio of the output change of the feedback $(\Delta \log (Y))$ and no-feedback locally analogous $\left(\Delta \log \left(Y_{N F}\right)\right)$ systems after a small perturbation $\left(\rho \rightarrow \rho^{\prime}\right)$. Left panel gives the formula for CoRa and the right panel shows a graphical interpretation of this quantity. (D) $\operatorname{CoRa}_{\theta \in \Theta}(\rho)$ for perturbations in $\rho$ can be calculated across a range of values of a chosen parameter $\theta \in \Theta$. This function gives insight as to potency of control for different values of the parameters. For example, one can specify a defined threshold $(\epsilon)$ for CoRa as an acceptable performance metric, and explore the parameter range for which the control is better than $\epsilon$. 
$(\Delta \log (Y))$ and its locally analogous system without feedback $\left(\Delta \log \left(Y_{N F}\right)\right), \operatorname{CoRa}_{\theta \in \Theta}(\rho)=\frac{\Delta \log (Y)}{\Delta \log \left(Y_{N F}\right)}$ (Fig. 1 C, the $\theta \in \Theta$ notation is added to indicate that CoRa can be computed for different parameters of the system). Being locally analogous, the two compared systems possess the same nonlinearities and saturations under the given parameter set $\Theta$. As a result, any differences in the response to a small perturbation are attributed to the effect of feedback (Supplementary Information). CoRa provides an easily interpretable assessment of how a system with feedback, positioned at the parameter set $\Theta$, fares compared to a no-feedback system when $\rho$ is perturbed. For instance, if $\operatorname{CoRa}_{\theta \in \Theta}(\rho) \in[0,1)$, the presence of the feedback reduces the effect of the perturbation compared to the locally analogous system without feedback, $\Delta \log (Y)<\Delta \log \left(Y_{N F}\right)$ (Fig. S1). When $\operatorname{CoRa}_{\theta \in \Theta}(\rho)=0$, the feedback endows the system with perfect adaptation $(\Delta \log (Y)=0)$, with the output returning exactly to the pre-perturbed state even in the continued presence of the perturbation. The value of $\operatorname{CoRa}_{\theta \in \Theta}(\rho)$ increases as the control effect decreases, and when $\operatorname{CoRa}_{\theta \in \Theta}(\rho)=1$, the feedback is ineffective as the output of the system with feedback becomes indistinguishable from that of the system without feedback (i.e. $\Delta \log (Y)=\Delta \log \left(Y_{N F}\right)$ ). This procedure can be repeated for any parameter set of interest. Specifically, we can compute CoRa for a range of values of the parameter $\theta \in \Theta$ while adjusting the constant input of the no-feedback system accordingly to ensure the mathematically controlled comparison in the sense we describe above. We therefore uniformly use the notation $\operatorname{CoRa}_{\theta \in \Theta}(\rho)$ to designate the CoRa function computed for a perturbation in $\rho$ when the system is positioned at some changing value of parameter $\theta \in \Theta$. $\operatorname{CoRa}_{\theta \in \Theta}(\rho)$ is therefore a representation of the capacity of feedback to mediate adaptation of the system's output to perturbations to the parameter $\rho$ for every value of $\theta$ considered. In this work, $\theta$ is limited to a change in an individual parameter.

Using CoRa to characterize negative feedback in a system architecture capable of perfect adaptation. We first tested CoRa on a well-established negative feedback control structure, the antithetic feedback motif, which can exhibit perfect adaptation to step disturbance inputs when connected to an arbitrarily complex biochemical network 2 (Fig. 2A). The antithetic motif is composed of two molecular species that annihilate each other through their mutual binding. One of the antithetic molecular species controls the input of a biochemical network and the other is produced by the output of the same network. If the antithetic molecules are only lost through the mutual annihilation event without individual degradation or dilution, this strategy is expected to generate a system with perfect adaptation to a step perturbation 22. Using CoRa to study this feedback motif, we recapitulate this result, showing that perfect adaptation is possible (Fig. 2). Interestingly, our analysis also reveals that relaxing the assumption of zero dilution and adding molecular details such as explicit accounting of the transitory molecule resulting from binding of the two antithetic molecules (complex $C$ in Fig. 2A) is sufficient to compromise perfect adaptation, often in non-trivial ways (Fig. 2 $2 \mathrm{~B}-\mathrm{C})$. For example, $\mathrm{CoRa}_{\mu_{Y} \in \Theta}\left(\mu_{Y}\right)\left(\mu_{Y}\right.$ is the synthesis rate of the output molecule $Y$ ) deviates from perfect adaptation value of 0 if dilution of antithetic molecules is assumed to occur individually at a small rate $\gamma=10^{-4} \mathrm{~min}^{-1}$ (Fig. 2B,D). This deviation from perfect adaptation occurs at low and high values of $\mu_{Y}$ (Fig. 2B; Supplementary Information). In a further elaboration of the circuit, when we consider the complex $C$ as a functional molecule that can influence the synthesis of the output molecule $Y$ until its removal from the system 11 (Fig. 22A), the feedback undergoes a dramatic failure in its ability to produce perfect adaptation after a specific threshold value of $\mu_{Y}$. This is evidenced by $\operatorname{CoRa}_{\mu_{Y} \in \Theta}\left(\mu_{Y}\right)$ shifting abruptly from almost zero to one (Fig. 2B,D). This is also the case for $\mathrm{CoRa}_{\eta_{-} \in \Theta}\left(\mu_{Y}\right)\left(\eta_{-}\right.$is degradation rate of complex $C$; Fig. $2 \mathrm{C}$ ). These conclusions are not unique to the particular controlled subsystem considered (Fig. S2), or the perturbation used (Fig. S3). Exploration of this phenomenology identified by CoRa reveals that this qualitative change in the feedback control results from saturation in the concentration of the complex $C$ (Supplementary Information; Fig. S4), an insight that would have been difficult without the computational observation of this behavior.

Using CoRa to compare different feedback control mechanisms. Any feedback control system can be analyzed using CoRa, providing a unifying framework under which different feedback mechanisms can be rigorously compared. In fact, we were able to rapidly analyze a large number of distinct feedback control motifs proposed in the literature 2, 4, 6, 7, 11, 13, 15 (Fig. 3, Supplementary Information). For comparison on equal footing, we considered each of these different negative feedback structures controlling the same simple biochemical subsystem. These investigations using CoRa generated a rich data-set to explore the properties of different molecular implementations associated with the same phenomenological macroscopic function -negative auto-regulation. For example, it was clear that specific molecular details of feedback generate distinct adaptation properties to the same perturbation. As only 

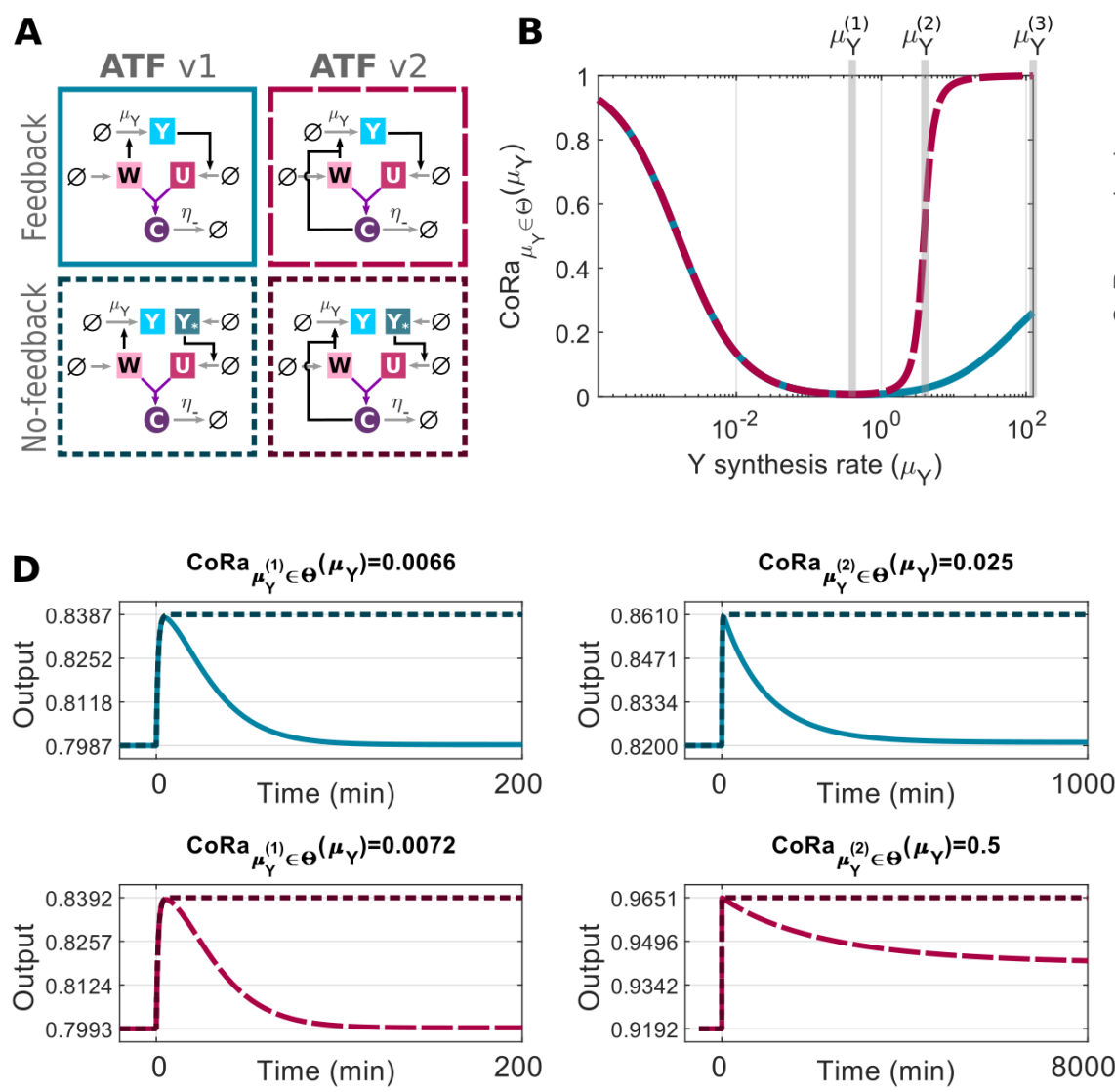
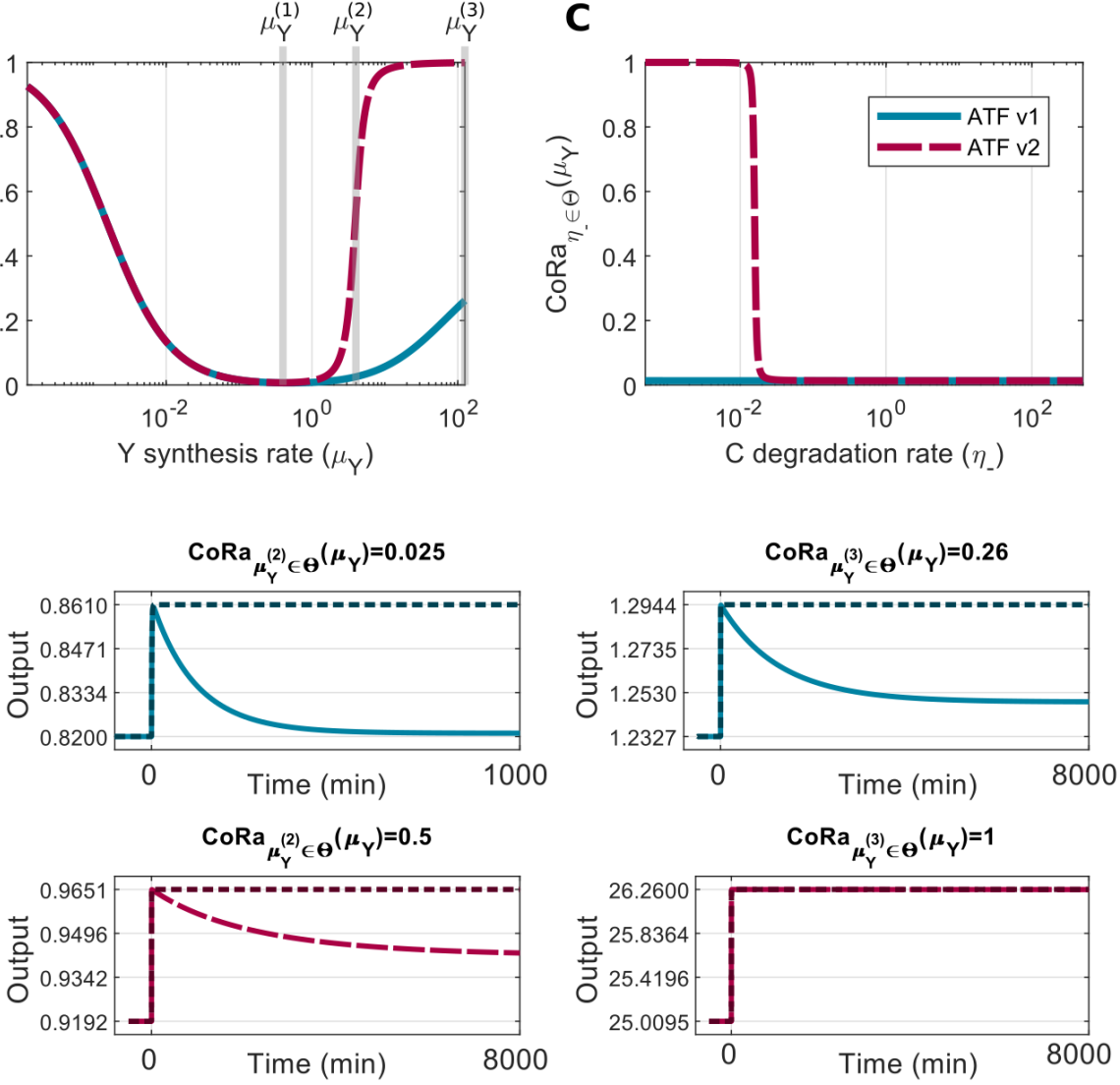

Figure 2. Characterizing the Antithetic Feedback Motif (ATF) using CoRa. (A) The ATF motif is composed by two molecules $(W, U)$ that bind and inactivate each other, forming a transitory complex $C$ which is then degraded with rate $\eta_{-}$; one antithetic molecule $W$ induces $Y$ synthesis (the controlled output, with rate $\mu_{Y}$ ), and $Y$ feeds back by inducing the synthesis of the other antithetic molecule, $U$. We consider two variations of the feedback structure. The first (ATF v1; blue continuous lines) is akin to the original ATF motif with the difference that the binding of $U$ and $W$ generates a complex $C$ that is explicitly modeled before it disappears through degradation at a rate $\eta_{-}$. In the second feedback structure (ATF v2; pink long-dash lines), the complex $C$ retains biological activity in influencing the production of $Y$ until it is degraded. This structure is inspired by the feedback implementation documented in $\mathrm{Ng}$ et al. [11. For each case, the associated locally analogous system without feedback (bottom box with dotted line) is shown. (B) CoRa computed following perturbations to $\mu_{Y}$, the synthesis rate of $Y$, as this parameter itself is varied. (C) CoRa computed following a perturbation to $\mu_{Y}$, the synthesis rate of $Y$, as the degradation rate of the complex $C\left(\eta_{-}\right)$is varied. (D) The output response of the ATF system (output is $Y$, blue continuous lines, v1, and pink long-dash lines, v2) and associated locally analogous systems without feedback (dark dash lines) as a function of time after a small perturbation occurs on $\mu_{Y}$ ( $\mu_{Y} \rightarrow \mu_{Y}^{\prime}$ at time zero). Plots are shown for three different $Y$ synthesis rate values $\mu_{Y}^{(i)}$ (highlighted in panel B with gray vertical lines). The resulting $\mathrm{CoRa}_{\mu_{\mathrm{x}}^{(\mathrm{i})} \in \Theta}\left(\mu_{Y}\right)$ value is shown as the plot title for each case. See Supplementary Information for equations and Table S1 for parameter values. 
one example, $\mathrm{CoRa}_{\mu_{\mathrm{Y}} \in \Theta}\left(\mu_{Y}\right)$ computed for all feedback strategies employing repression of synthesis modelled using a standard Michaelis-Menten repression function (Fig. 3G-I) displayed a limit of $\operatorname{CoRa}_{\theta \in \Theta}\left(\mu_{Y}\right) \geq 0.5$. This behavior relates to the inevitable saturation of the repression function (see Supplementary Information for an example of an analytical treatment of this limit). A notable exception to this limit occurred for the "brink motif" feedback strategy, a motif that combines antithetic molecular sequestration with an activation-deactivation enzymatic cycle to produce a tuneable ultra-sensitive response [13] (Fig. 3K ). These patterns that were computationally pinpointed by the CoRa analysis prompted the hypothesis that adding ultra-sensitivity to motifs with Michaelis-Menten synthesis repression might alleviate the limit of their adaptive behaviors. Using CoRa, we tested this hypothesis by adding a Hill coefficient larger than 1 to the Michaelis-Menten function in different strategies. By increasing the system ultrasensitivity with the Hill coefficient, the lower bound of the CoRa curve decreased in all cases, indicating improved adaptation capabilities of the control loops (Fig. S5A-C). Furthermore, increasing the ultrasensitivity of the brink motif itself by increasing the deactivation rate in its enzymatic cycle 13] improved its ability to adapt (Fig. S5D). These results strongly suggest that feedback strategies based on Michaeliean repression of synthesis are severely limited in their capacity for homeostasis, but can be improved using ultra-sensitive components. In this case, CoRa was used as a computational hypothesis generator about this general principle, which was then confirmed through further computational and analytical investigations. Finally, identification of strategies for improving feedback performance can be automated by embedding CoRa into an optimization framework in which the parameters of the feedback are iteratively changed to generate a desirable CoRa curve (Fig. S6). This optimization procedure can help uncover the parameter constraints that are needed for adaptation given the specific biochemical feedback structure in a system. It can also help in the design of de novo synthetic feedback structures in cells.

Discussion. A framework for the systematic evaluation and comparison of biochemical feedback control systems is essential for understanding the general principles of biological homeostasis. While many methods exist for the evaluation of technological feedback systems, understanding the principles of biological adaptation mediated through feedback poses its unique challenges, including distinct mathematical properties of the biological substrate.

Importantly, the nature of biological organization with extensive coupling of parameters and processes makes the extraction of engineering-centric quantities needed for traditional analyses of feedback quantities, such as setpoints and regulation errors, challenging. Debate about whether these quantities are defined for biological systems has a long history and no concrete resolution [12. One advantage of CoRa is that it does not make any assumptions about the existence of such quantities, replacing this debate with a comparison to a system that would have evolved identically but without the feedback structure. Another advantage of CoRa is that it is agnostic to the complexity of the system. While we have only used a simple system to illustrate the properties of CoRa, extending analysis to more complex systems was straightforward (Fig. S2). We have also used CoRa to assess feedback-mediated adaptation for only one perturbation as a function of one model parameter. However, it should be easy to see that a multi-dimensional CoRa for simultaneous perturbations or many concurrent parameter changes is easily computable. We expect, however, that new methods would be needed to analyze the resulting multi-dimensional data into coherent principles.

Finally, the concept of CoRa should be easily extendable to assessing the quantitative contribution of feedback to important properties other than the steady-state response to perturbation. These might, for example, include the role of feedback in the dynamic response of a system or its response to stochastic fluctuations. As such, CoRa represents a flexible framework that is poised to catalyze fast progress in our understanding of the many roles that feedback control plays in biological organization.

\section{SUPPLEMENTARY MATERIALS}

\section{Supplementary Figures: Fig S1-S6}

Supplementary Tables: Table S1-S2

Supplementary Text: Section S1-S5

Supplementary Code: https://github.com/mgschiavon/CoRa/releases/tag/v1.0 

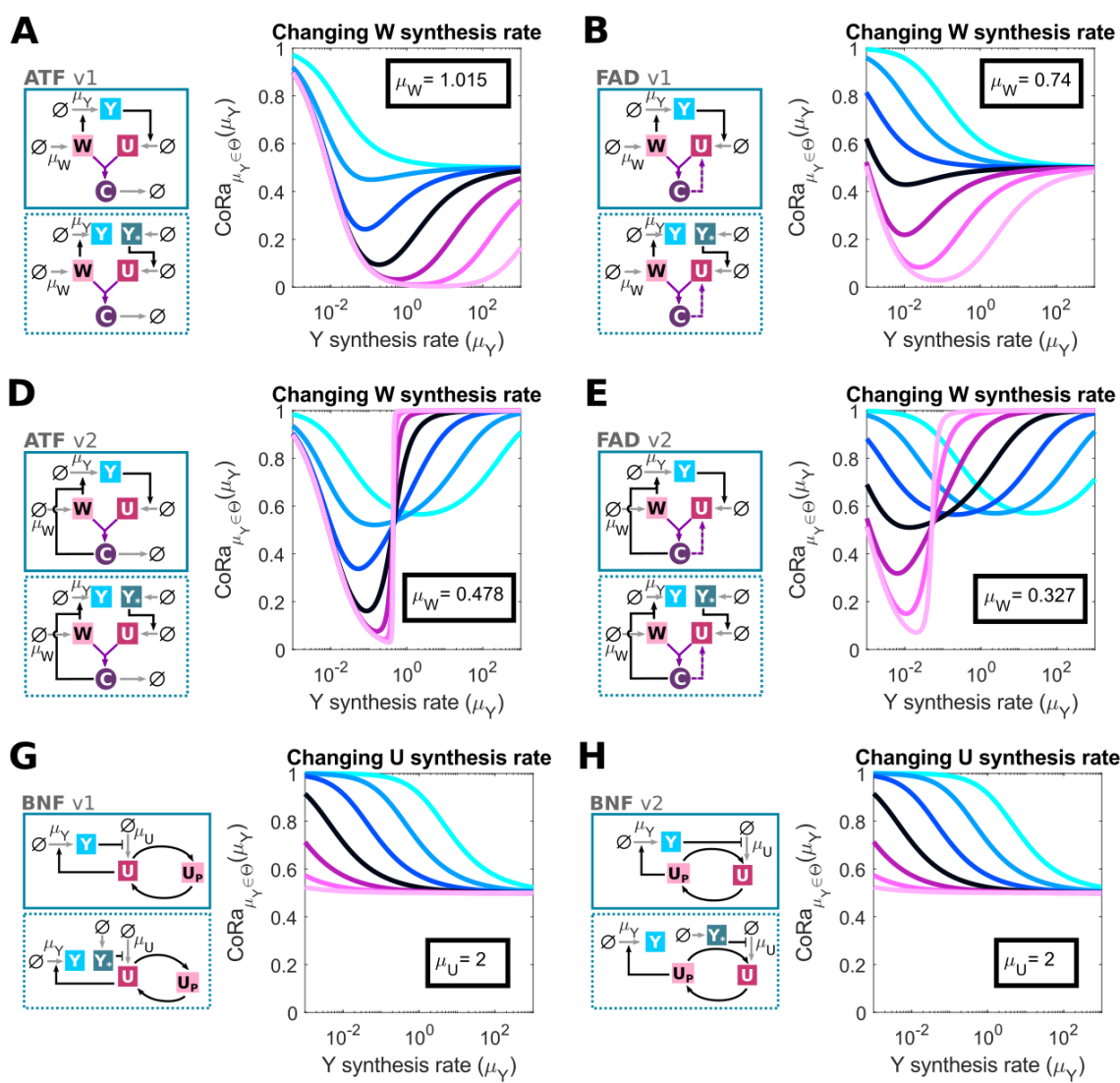
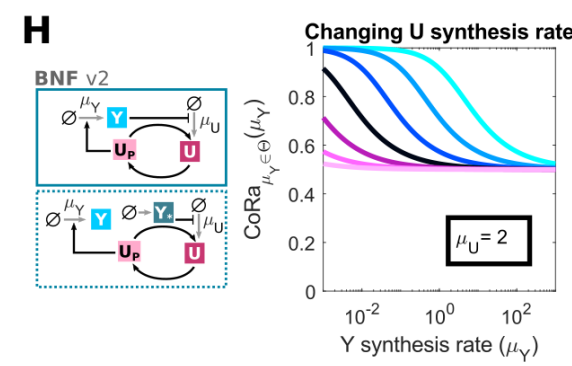
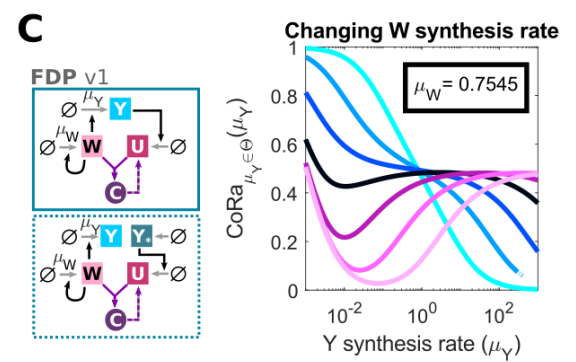

$\mathbf{F}$
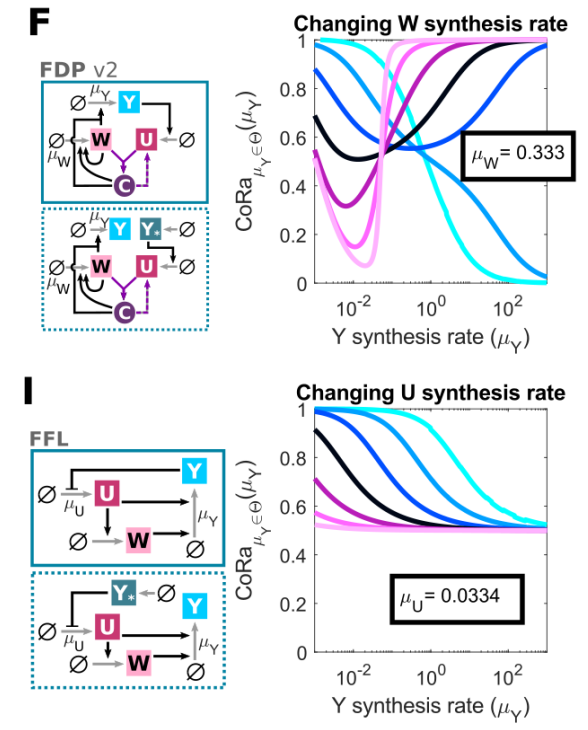

J
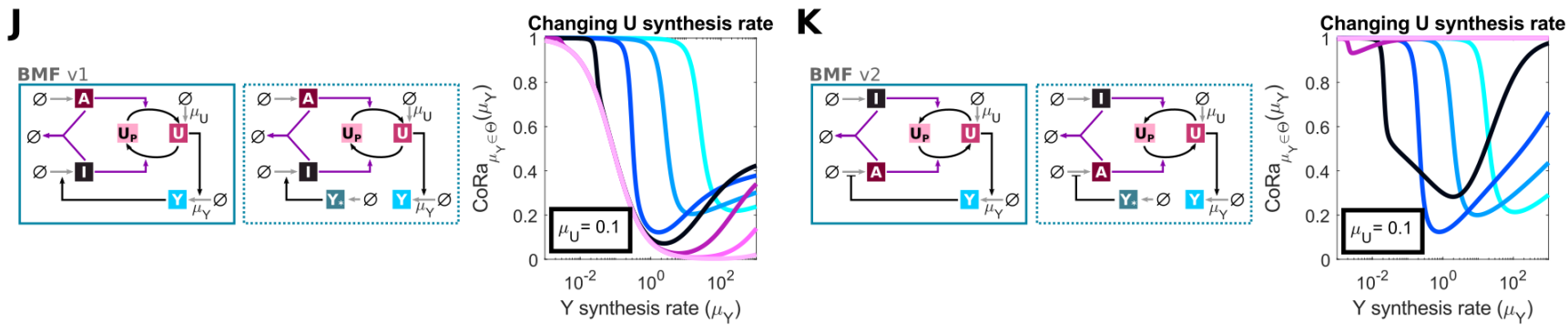

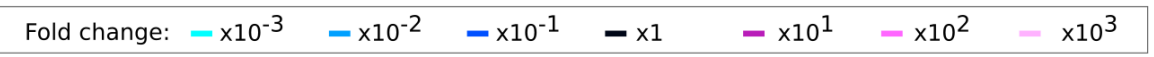

Figure 3. CoRa provides a unifying framework to compare different feedback control architectures. (A-K) Different feedback motifs, with different levels of complexity, can be directly compared using the CoRa function. In each case, the diagrams of the feedback system (in box with continuous line) and its associated locally analogous system without feedback (in box with dotted line) are shown. $\mathrm{CoRa}_{\mu_{\mathrm{Y}} \in \Theta}\left(\mu_{Y}\right)$ is computed for 7 different values of a given parameter that is also varied in addition to $\mu_{Y}$. The identity and nominal value of the varied parameter (either $\mu_{W}$ the $W$ synthetic rate, or $\mu_{U}$ the $U$ synthesis rate) is indicated on every plot, and how it is varied is shown at the bottom of the figure with appropriate color-coding information. Different mechanisms show different signature behaviors; particularly, for ATF-like architectures, a complex $C$ of the two antithetic molecules that retains activity results in an abrupt loss of control (D-F) compared to the analogous motifs with an inactive complex (A-C). The Michaelis-Menten function describing feedback through repression of synthesis imposes a limit on the efficiency of feedback (G-I), except in the presence of ultrasensitivity (K). ATF: Antithetic Feedback, FAD: Feedback by Active Degradation, FDP: Feedback by Active Degradation + Positive Feedback, BNF: Buffering + Negative Feedback, FFL: Negative Feedback + Feed-forward loop, and BMF: Brinf Motif. In all cases, the controlled subsystem parameters are identical, and the output steady state $Y \approx 10 \mathrm{nM}$ for the " $\times 1$ " case (black line) and $\mu_{Y}=1 \mathrm{~min}^{-1}$. See Supplementary Information for equations and Table S1 for parameter values. 


\section{Acknowledgments}

The authors would like to thank the members of the El-Samad lab for useful comments and discussion on the manuscript. This work was supported by the Chan-Zuckerberg Biohub and the Defense Advanced Research Projects Agency, Contract No. HR0011-16-2-0045 to H.E.-S. The content and information does not necessarily reflect the position or the policy of the government, and no official endorsement should be inferred. H.E.-S. is a Chan-Zuckerberg investigator.

\section{References}

1. R. Alves and M. A. Savageau. Extending the method of mathematically controlled comparison to include numerical comparisons. Bioinformatics, 16(9):786-798, sep 2000.

2. C. Briat, A. Gupta, and M. Khammash. Antithetic Integral Feedback Ensures Robust Perfect Adaptation in Noisy Biomolecular Networks. Cell Systems, 2(1):15-26, jan 2016.

3. D. Del Vecchio and R. M. Murray. Biomolecular Feedback Systems. Princeton University Press, 2014.

4. T. Drengstig, X. Y. Ni, K. Thorsen, I. W. Jolma, and P. Ruoff. Robust Adaptation and Homeostasis by Autocatalysis. The Journal of Physical Chemistry B, 116(18):5355-5363, may 2012.

5. H. El-Samad, J. P. Goff, and M. Khammash. Calcium homeostasis and parturient hypocalcemia: an integral feedback perspective. Journal of theoretical biology, 214(1):17-29, jan 2002.

6. E. J. Hancock, J. Ang, A. Papachristodoulou, and G.-B. Stan. The Interplay between Feedback and Buffering in Cellular Homeostasis. Cell Systems, 5(5):498-508.e23, nov 2017.

7. A. W. Harris, J. A. Dolan, C. L. Kelly, J. Anderson, and A. Papachristodoulou. Designing Genetic Feedback Controllers. IEEE Transactions on Biomedical Circuits and Systems, 9(4):475-484, 2015.

8. S. Hohmann. Osmotic stress signaling and osmoadaptation in yeasts. Microbiology and Molecular Biology Reviews, 66(2):300-372, 2002.

9. A. J. Miller and S. J. Smith. Cytosolic Nitrate Ion Homeostasis: Could it Have a Role in Sensing Nitrogen Status? Annals of Botany, 101(4):485-489, 122008.

10. R. W. Moncrieff. Olfactory adaptation and odour likeness. The Journal of physiology, 133(2):301-16, aug 1956.

11. A. H. Ng, T. H. Nguyen, M. Gómez-Schiavon, G. Dods, R. A. Langan, S. E. Boyken, J. A. Samson, L. M. Waldburger, J. E. Dueber, D. Baker, and H. El-Samad. Modular and tunable biological feedback control using a de novo protein switch. Nature, 572(7768):265-269, aug 2019.

12. G. B. Risvoll, K. Thorsen, P. Ruoff, and T. Drengstig. Variable setpoint as a relaxing component in physiological control. Physiological Reports, 5(17):1-15, 2017.

13. C. C. Samaniego and E. Franco. Ultrasensitive molecular controllers for quasi-integral feedback. bioRxiv, pages $1-27,2018$.

14. R. Thomas and R. d'Ari. Biological Feedback. CRC Press, Inc., 1990.

15. F. Xiao and J. C. Doyle. Robust Perfect Adaptation in Biomolecular Reaction Networks. bioRxiv, 2018.

16. T.-M. Yi, Y. Huang, M. I. Simon, and J. Doyle. Robust perfect adaptation in bacterial chemotaxis through integral feedback control. Proceedings of the National Academy of Sciences, 97(9):4649-4653, apr 2000. 